\title{
Material-induced shunts in multicrystalline silicon solar cells
}

\author{
(C) O. Breitenstein ${ }^{\pi}$, J. Bauer, J.P. Rakotoniaina ${ }^{1}$
}

Max Planck Institute of Microstructure Physics, Weinberg 2, D-06120 Halle, Germany

(Получена 12 сентября 2006 г. Принята к печати 3 октября 2006 г.)

By applying lock-in thermography imaging, light-beam induced current imaging, electron-beam induced current imaging at different stages of sample preparation, and infrared light microscopy in transmission mode, the physical nature of the dominant material-induced shunts in multicrystalline solar cells made from $p$-type silicon material has been investigated. It turns out that these shunts are due to silicon carbide ( $\mathrm{SiC})$ filaments, which are growing preferentially in grain boundaries and are crossing the whole cell. These filaments are highly $n$-type doped, like the emitter layer at the surface of the cells. They are electrically connected both with the emitter and with the back contact, thereby producing internal shunts in the solar cell.

PACS: 84.60.Jt, 87.63.Hg, 87.64.Ee, 87.64.Rr

\section{Introduction}

Block-cast multicrystalline (m.c.) silicon is today's dominant material for producing solar cells for direct conversion of solar radiation to electrical energy. The world production volume of solar modules based on m.c. material exceeded $1 \mathrm{GW}$ installed power in 2005 with exponential growth tendency, which points to the high economic impotrance of this material. Multicrystalline $p$-type silicon is cast in form of several $100 \mathrm{~kg}$ sized blocks, which slowly crystallize from bottom to top, thus yielding relatively large $\mathrm{mm}$ - to $\mathrm{cm}$-sized crystallites. These blocks are cut into bricks, which are wire-sawed into wafers for solar cell production. The casting crucibles consist from quartz material, which is covered with $\mathrm{Si}_{3} \mathrm{~N}_{4}$ material. For melting the silicon ingot material, they are heated by graphite heaters in an inert gas atmosphere. The dominant impurities in this material are carbon, nitrogen, and oxygen.

Solar cells made from m.c. material regularly suffer from ,shunts“, which are internal short-circuits degrading the parameters of the cells and often even preventing their applicability. Some of these shunts are due to technological faults, but very often they are material-induced. In earlier investigations, silicon carbide particles were suspected to be responsible for these shunts. For the conduction mechanism, an electrostatic inversion layer around $\mathrm{SiC}$ precipitates was assumed to cross the cells [1]. In this contribution, a systematic investigation of the physical nature of materialinduced shunts in m.c. silicon solar cells is presented. It will turn out that the shunts consist not from $\mathrm{SiC}$ particles but from $\mathrm{SiC}$ filaments crossing the cells, which are highly $n$-conducting. Hence, the shunt current is flowing not in an inversion layer around the precipitates, as suspected earlier, but rather within the volume of the precipitates.

\footnotetext{
${ }^{1}$ Present adress: Q-Cells AG, D-06766 Thalheim, Germany.

ฯ E-mail: breiten@mpi-halle.mpg.de
}

\section{Experimental results}

\subsection{Lock-in thermography, light-beam induced current and electron-beam induced current imaging}

Lock-in thermography (LIT) [2-4] is the most successful technique to image shunts in solar cells. It this technique, the cell is imaged by a high-sensitive infrared (IR) thermocamera, a pulsed bias is applied to the cell, and the IR images are processed and averaged over a certain acquisition time in a computer according to the lock-in principle to yield the amplitude image of the local surface temperature modulation. After some minutes of acquisition and averaging, temperature modulations below $0.1 \mathrm{mK}$ can be detected by LIT. In the positions of shunts, the dissipated heat shows a maximum, leading to a bright spot in the LIT image. By comparing images measured under forward and reverse bias, the conductivity type of a shunt can be checked: only if a shunt shows an ohmic (linear) currentvoltage $(I-V)$ characteristic, its LIT image is the same under forward and under reverse bias.

Light beam-induced current (LBIC) [1,5] is the standard technique to image the homogeneity of the short circuit current of solar cells. A laser spot is focused to the cell surface, and the induced current is measured under short circuit conditions. As the cell is raster-scanned, an image of the local short circuit current is formed. In electron beam-induced current (EBIC) imaging [6] basically the same is done, except that a scanning focused electron beam is used instead of the fixed laser beam and mechanical sample scanning. EBIC shows a considerably better spatial resolution than IBIC $(<1 \mu \mathrm{m}$ for EBIC, $<(10-20) \mu \mathrm{m}$ for LBIC). It had been shown [1] that SiC-induced shunts become visible in LBIC (and EBIC) not by the usual recombination contrast but by the „resistive current divider" contrast mechanism, which drains some part of the locally induced current by the shunt, if the shunt resistance comes into the order of the series resistance to the current amplifier (see next section). 

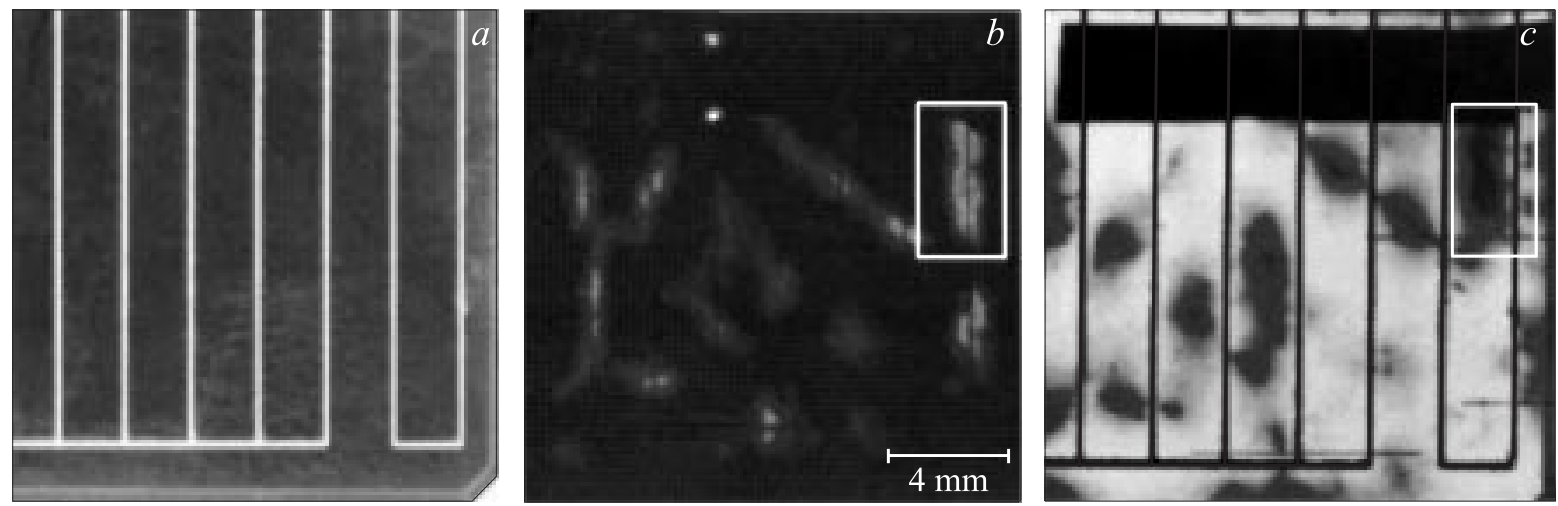

Figure 1. Optical image $(a)$, lock-in thermogram $(b)$, and LBIC image $(c)$ of a region in the solar cell containing material-induced shunts.
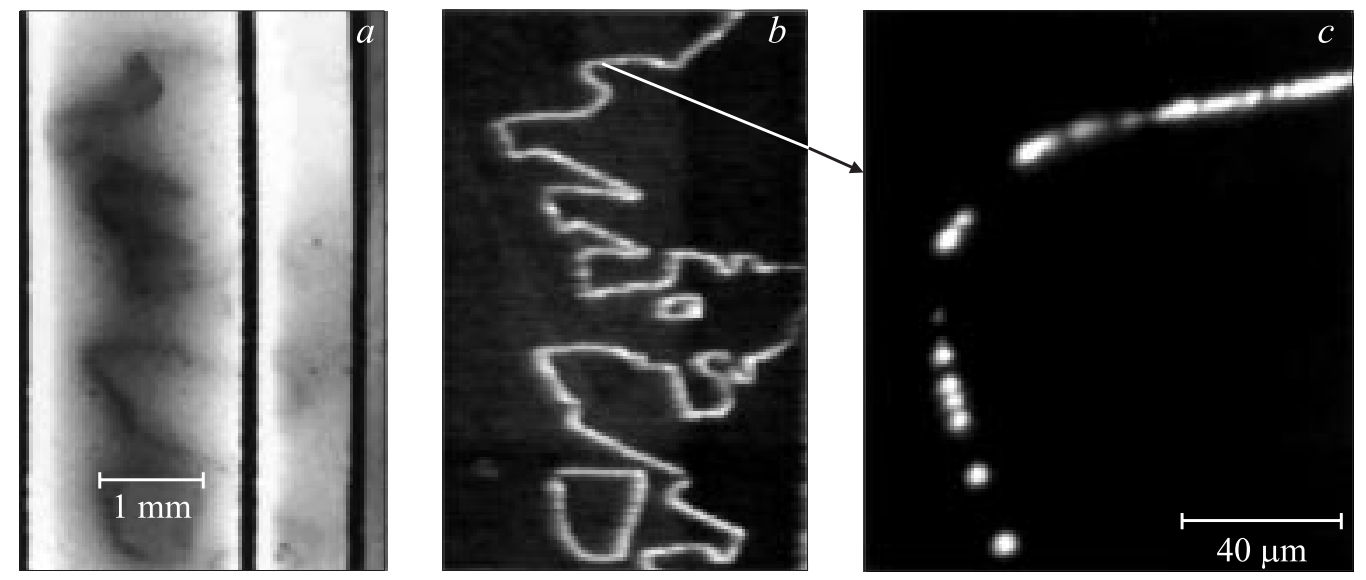

Figure 2. EBIC image $(a)$ of the region framed in Fig. 1, backside EBIC image (mirrored) of the same region $(b)$, and detail from $(b)$ in the indicated region $(c)$. The acceleration voltage was $10 \mathrm{kV}$.

Fig. 1 shows the optical image $(a)$, the LIT image $(b)$, and the LBIC image $(c)$ of the region in the multicrystalline silicon solar cell, which contains material-induced shunts. The shunting regions are shown in the LIT image as bright regions and in the LBIC image as dark regions. The LIT image $(b)$ was taken under forward bias condition, but the same image appeared under reverse bias, hence these shunts show a linear $I-V$ characteristic. A general correspondence can be seen between LIT and LBIC, but there are also some apparent discrepancies. So the center of the dominant shunt in the framed region seems to be below the grid line in the LIT image, but between the grid lines in the LBIC image.

In order to obtain a better spatial resolution, EBIC imaging was performed in the framed region, with the result shown in Fig. 2, $a$. Here we see that the shunting region actually consists from lines, which are in the positions of some grain boundaries of the material. Also in this image the EBIC contrast of the defects is strongest between the grid lines and weakest close to the grid lines. After this investigation, the back contact of this cell was polished away to the silicon base. Then the cell was contacted between the emitter and a rubbed-on $\mathrm{Ga} / \mathrm{In}$ contact to the base material, and the sample was imaged by EBIC from its backside, with the electron beam hitting the bare base material. For a better comparison with the other EBIC images, these backside EBIC images are displayed horizontally mirrored. Fig. 2 shows that the dark lines in the frontside EBIC image $(a)$ correspond to bright lines in backside EBIC image (b). While the frontside EBIC contrast was quite cloudy, the backside EBIC contrast is very sharp. Indeed, if this region is imaged with high magnification $(c)$, the bright lines are shown to consist from single bright spots, which show a collection efficiency close to 1 . These spots are all lying in grain boundaries. There is no influence of the grid lines anymore in the backside EBIC images.

\subsection{Interpretation of the images}

An unifying explanation of all experimental observations shown in the previous section can be made by assuming that the defects, which are responsible for the shunts, are rows of parallel running $n$-conducting channels crossing the cell in the position of grain boundaries, thereby shunting the emitter against the back contact. As it had been 


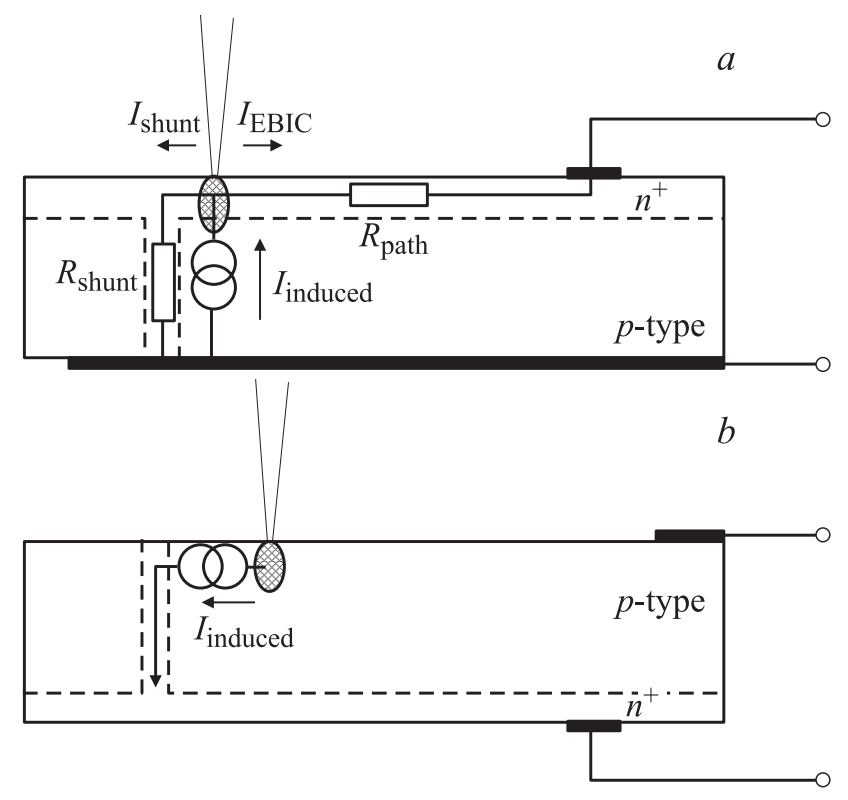

Figure 3. Schematic of frontside EBIC imaging ( $a$, see [1]) and backside EBIC imaging $(b)$ of an $n$-type channel.

outlined earlier [1], under this condition the defect contrast for frontside EBIC imaging is not due to recombination at the defects but due to a current splitting effect, which is described in Fig. 3, $a$. Since the EBIC amplifier represents a short circuit, the locally induced current $I_{\text {induced }}$ splits into two parts, $I_{\text {shunt }}$ and $I_{\mathrm{EBIC}}$, according to $[1]$

$$
I_{\text {EBIC }}=I_{\text {induced }} \frac{R_{\text {shunt }}}{R_{\text {path }}+R_{\text {shunt }}} .
$$

Note that, for a certain distance between the generation region and the shunt, $R_{\text {shunt }}$ also contains a distancedependent contribution from the emitter resistance. Depending on the distance between generation region and shunt, and on the distance between generation region and grid line, $R_{\text {shunt }}$ and $R_{\text {path }}$ are varying. If a shunt is lying close to a grid line, $R_{\text {path }}$ is very low and $I_{\mathrm{EBIC}}=I_{\text {induced }}$ holds. This is the reason why in LBIC (Fig. 1,c) and in frontside EBIC (Fig. 2,a) the shunts between the grid lines are appearing with a stronger contrast than the shunts close to the grid lines. For the thermal LIT signal the opposite argument holds: here the shunt current is the larger, the lower the series resistance to the shunt is. Therefore, in the LIT image Fig. 1, $b$, the shunts below the grid lines are more pronounced than between them. This explains the apparent discrepancy between Fig. 1, $b$ and $c$ in the marked region.

If the back contact is removed by mechanical polishing, these shunts should be disabled, since the $n$-type cannels are lying free in the material now. Indeed, after polishing away the back contact, the bright thermographic contrast of Fig. 1, $b$ disappeared, and the shunt resistance of the device increased by several orders of magnitude. Now, since the local $n$-regions are lying at the free surface, they may collect minority carriers in backside EBIC imaging, as schematically shown in Fig. 3, b. The polished surface show a very high recombination rate, and the penetration depth of the electron beam is only a few $\mu \mathrm{m}$ at the used acceleration voltage of $10 \mathrm{kV}$. Therefore, if the generation region is away from the $n$-type channels, the collection probability is very low, in spite of the long diffusion length in the base material. Only if the generation region is close to an $n$-type channel, most of the generated minority carriers are collected. This is the reason for the very good spatial resolution in the backside LBIC images as demonstrated in Fig. 2, c. Since these are very small currents (in the $\mu \mathrm{A}$ range), any series resistance does not play a role here. Therefore the positions of the grid lines do not influence the backside LBIC images, as had been observed in Fig. $2, b$.

\subsection{Origin of the $n$-type channels}

After the backside EBIC images have been made, the sample was etched for a few seconds in $\mathrm{HF}-\mathrm{HNO}_{3}$, whereby the frontside emitter was protected by black wax. After removing the wax, the sample was subjected again to scanning electron microscope inspection in secondary electron (SE) and backside EBIC mode. Now, in the shunt positions in the grain boundaries, rows of several $\mu \mathrm{m}$ thick filaments were sticking out of the surface of the sample, as Fig. 4, $a$ shows. According to Fig. 4, $b$, the EBIC signal was still present when the beam hitted the Si material around the
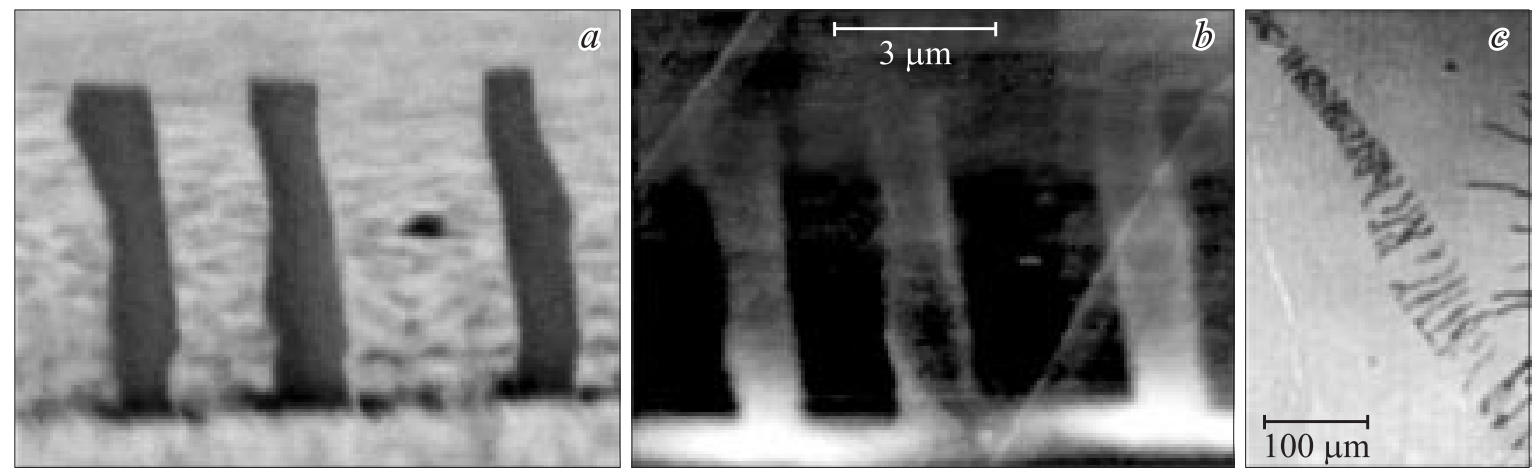

Figure 4. Secondary electron image $(a)$ and backside EBIC image $(b)$ of SiC filaments, sticking out of the Si surface in a shunt position. $c$ - IR transmission light microscopy image of $\mathrm{SiC}$ filaments crossing a $\mathrm{Si}$ wafer in the position of a grain boundary. 
filaments. Electron dispersive $X$-ray spectroscopy (EDX) analysis has revealed that these filaments consist from $\mathrm{SiC}$, which is not attacked by the $\mathrm{HF}-\mathrm{HNO}_{3}$ etchant.

After the solar cell was mechanically polished at both sides, it can be imaged in a light microscope in transmission mode in near-infrared region by using a CCD-camera [7]. Fig. 4, $c$ shows such image in a typical shunt region along a grain boundary. We see a row of more or less parallel running filaments in a bent plane, sometimes branching, which are the $\mathrm{SiC}$ filaments in the grain boundary. Also TEM investigations have revealed by energy dispersive $X$-ray $(\mathrm{EDX})$ and diffraction analysis that these filaments consist from cubic SiC [7]. Obviously, these filaments are growing over a length of several $\mathrm{mm}$ preferentially within grain boundaries in growth direction of the material. Most recent electrical investigations on single filaments have shown [8] that the $\mathrm{SiC}$ material is $n$-conducting, most probably due to incorporation of nitrogen, with an electron concentration of several $10^{18} \mathrm{~cm}^{-3}$. Nitrogen is the dominant shallow donor in $\mathrm{SiC}$ and is, together with carbon and oxygen, one of impurities with highest concentration in cast solar silicon material.

\section{Conclusion}

In contrast to earlier speculations $[1,7]$, the defects being responsible for material-induced shunts in cast silicon solar cells are not $\mathrm{SiC}$ particles but rather $\mathrm{SiC}$ filaments crossing the bulk of the cell. They are $n$-conducting and yield a heterojunction to the $p$-type silicon base, which is switched in parallel to the emitter and may collect minority carriers. However, if these filaments are electrically connected to the back contact, they produce shunts.

There are basically two ways to prevent the appearance of these shunts, which are the reduction of the carbon concentration in the material and the blocking of the shunt path. The casting of the silicon blocks appears usually in quartz crucibles, which are standing in a graphite mold and are internally covered with a $\mathrm{Si}_{3} \mathrm{~N}_{4}$ layer. The whole apparatus is heated by graphite heaters and is protected by inert gas (argon). Even lowest concentrations of oxygen or water in the inert gas may lead to oxidation of the graphite heaters producing $\mathrm{CO}$ and $\mathrm{CO}_{2}$, which easily dissolves into the liquid silicon. This mechanism and the carbon content in the ingots are probably the major sources of carbon in a block-cast material. An alternative way to prevent SiC-related shunting is to block the shunt path. Novel ligh-efficiency solar cell technologies are working with an dielectric layer at the backside of the cells, which contains a pattern of point-contacts [9]. Thus, if the total back contact area reduces e.g. by a factor of 100 , also the probability to have material-induced shunts reduces by this factor. Hence, solar cells with point contact backside should be much less affected by material-induced shunting than present full-area back-contacted cells are.
This work was supportet by the German Federal Ministry of the Environment (BMU) under contract No. 0329846D (ASIS). The authors are grateful to Deutsche Solar and Photowatt for their cooperation.

\section{References}

[1] A. Kaminski, O. Breitenstein, J.P. Boyeaux, J.P. Rakotoniaina, A. Laugier. J. Phys.: Condens. Matter, 16, 9 (2004).

[2] S. Huth, O. Breitenstein, A. Huber, D. Dantz, U. Lambert, F. Altmann. Sol. St. Phenomena, 82-84, 741 (2002).

[3] O. Breitenstein, M. Langenkamp. Lock-in Thermography Basics and Applications to Functional Diagnostics of Electronic Components (Springer, Heidelberg-Berlin, 2003) ISBN 3-540-43439-9.

[4] O. Breitenstein, J.P. Rakotoniaina, M.H. Al Rifai, M. Werner. Progr. Photovolt. Res. Appl., 12, 529 (2004).

[5] J. Marek. J. Appl. Phys., 55, 1621 (1984).

[6] SEM Microcharacterization of Semiconductors, ed by D.B. Holt, D.C. Joy (AP, London, 1989).

[7] J.P. Rakotoniaina, S. Neve, M. Werner, O. Breitenstein. Proc. $P V$ in Europe: From PV Technology to Energy Solutions. Conference and Exhibition (Rome, 2002) p. 24.

[8] J. Bauer. Diploma Thesis (2006) at Martin-Luther-University Halle, Germany (in German). To be published at EDS conference 2006 (Halle, Germany).

[9] A. Grohe, U. Zastrow, D. Meertens, L. Houben, W. Brendle, G. Bilger, E. Schneiderlöchler, S.W. Glunz, R. Preu, G.P. Willeke. Proc. 20th Eur. Photovoltaic Solar Energy Conf. (Barcelona, 2005) p. 1158.

Редактор Л.В. Шаронова 